\title{
EFECTO DE LA HUMEDAD RELATIVA SOBRE LA GERMINACIÓN DE LAS ESPORAS DE Beauveria bassiana Y LA PATOGENICIDAD A LA BROCA DEL CAFÉ Hypothenemus hampei
}

\section{EFFECT OF ENVIRONMENTAL MOISTURE ON THE GERMINATION OF Beauveria bassiana SPORES AND THEIR PATHOGENICITY AGAINST THE COFFEE BERRY BORER}

\author{
Paula F. Arrubla M. ${ }^{1}$; Mauricio Cárdenas R. ${ }^{2}$ Francisco J. Posada F. ${ }^{3}$
}

\begin{abstract}
${ }^{1}$ Bacterióloga. Universidad Católica de Manizales. Centro Nacional de Investigaciones de Café, Cenicafé, Chinchiná, Caldas, Colombia. Sede Plan alto, km. 4 vía Chinchiná- Manizales. Chinchiná (Caldas) - Colombia. ${ }^{2}$ Diseñador Industrial. Programa jóvenes investigadores Convenio Colciencias - Cenicafé. Centro Nacional de Investigaciones de Café, Cenicafé, Chinchiná, Caldas, Colombia. Sede Plan alto, km. 4 vía Chinchiná-Manizales.Chinchiná (Caldas) - Colombia.Bacterióloga. ${ }^{3}$ Ing. Agrónomo. Entomólogo Ph.D. IPM-Perennial Crop Protection consultant. E-mail: fjavierposada@hotmail.com
\end{abstract}

Rev. U..D.C.A Actual. \& Div. Cient. 13(1): 67-76, 2010

\section{RESUMEN}

Este trabajo tuvo como objetivo evaluar la concentración, la pureza, la germinación y la patogenicidad sobre la broca del café de las esporas del hongo Beauveria bassiana en polvo, después de ser expuestas a los niveles de humedad de 99, 75, 65 y 39\%, que se obtuvieron utilizando soluciones saturadas mantenidas en cámaras húmedas, a una temperatura constante de $24^{\circ} \mathrm{C}$. Las evaluaciones del efecto de la humedad sobre las esporas, se realizaron luego de 1, 10 y 20 días de expuestas, a los niveles de humedad correspondientes. En los resultados, se obtuvo que la concentración, la pureza y la patogenicidad de las esporas, a través de los tiempos de exposición a los diferentes niveles de humedad, no presentaron marcada variación durante el tiempo de evaluación. La patogenicidad, se conservó con valores superiores a $86,7 \%$ en todos los niveles de humedad y tiempos de evaluación. La germinación fue la variable, que presentó la mayor variación y disminuyó, a través del tiempo: entre 88,9 a $82,1 \%$, luego de un día, entre 79 y $68 \%$, después de diez días y entre 62,2 a $56,2 \%$, posterior a los 20 días. La germinación para los diferentes niveles de humedad solo presentó diferencias significativas dentro de los tiempos de evaluación de 1 y 10 días.

Palabras clave: Hongo, patogenicidad, germinación, pureza, concentración, Hypothenemus hampei, café.

\section{SUMMARY}

The aim of this study was to evaluate the concentration, purity, germination and pathogenicity of Beauveria bassiana spores against the coffee berry borer, after being exposed to humidity levels of 99, 75, 65 and 39\%, which were obtained using saturated solutions and kept in humid chambers at a constant temperature of 24 ${ }^{\circ} \mathrm{C}$. Evaluations of the effect of moisture on the spores were carried out after 1,10 and 20 days of exposure. The results showed that the concentration, purity and pathogenicity of the spores through exposition times at 
different humidity levels did not present great variation during the time of evaluation. The pathogenicity remained above $86.7 \%$ at all humidity levels and evaluation times. The germination was the variable that presented the greatest variation and decreased over time: from 88.9 to $82.1 \%$ after one day, from 79 and $68 \%$ after ten days and from 62.2 to $56.2 \%$ after 20 days. The germination at different moisture levels showed significant differences only within the evaluation times of one and ten days.

Key words: Fungus, pathogenicity, germination, purity, concentration, Hypothemnmus hampei, coffee.

\section{INTRODUCCIÓN}

Dentro de las diferentes áreas de investigación sobre hongos entomopatógenos, la prolongación de la viabilidad de las esporas en almacenamiento recibe gran atención, como componente de la calidad y requisito para la comercialización de las formulaciones de estos agentes de control biológico (Bateman et al. 1993; Lisansky \& Coombs, 1994; Moore et al. 1995, Marquez \& Alves, 1996). Para alcanzar este objetivo, las esporas se cosechan y se conservan con bajos niveles de humedad y se almacenan a bajas temperaturas (Moore et al. 1995). Sin embargo, existe un gran desconocimiento del efecto de los factores ambientales sobre las esporas de los hongos, luego de aplicadas en el campo, especialmente, de la humedad y la temperatura, sobre la germinación y la patogenicidad. El efecto de la radiación solar ha recibido más atención y se conoce que tiene efecto deletéreo sobre la viabilidad de las esporas (Moore et al. 1993; Vélez \& Montoya, 1993; Inglis et al. 1995).

De los hongos entomopatógenos recién producidos o formulados, se conoce que las esporas inician la germinación cuando están en presencia de altos niveles de humedad relativa o agua libre (McCoy et al. 1988); sin embargo, existe evidencia que pueden germinar a bajos niveles de humedad cuando está presente el insecto hospedante (Ferron, 1978). En relación con la temperatura, se conoce que la sobrevivencia de las esporas se logra entre 20 y $30^{\circ} \mathrm{C}$. Si las esporas se exponen a temperaturas altas, alrededor de $45^{\circ} \mathrm{C}$, se puede presentar atraso de la germinación y no reducción en el número de esporas que germinan (Moore et al. 1995). La velocidad de germinación de las esporas puede tener ventajas en la infección de los hospedantes en campo, dependiendo del tiempo en que entran en contacto con el insecto contra el que va dirigida la aplicación (Posada E Vega, 2005).

Vélez (1997) encontró que después de asperjar esporas de $B$. bassiana formuladas en aceite o agua sobre el follaje de árboles de café era posible recuperar, a los 14 días, 50.000 unidades formadores de colonias del hongo. Estos resultados indican que de presentarse poblaciones de la broca y entrar en contacto con el follaje tratado con esporas es posible que ocurra la infección. Esto ha sido demostrado en bioensayo asperjando hojas de café en laboratorio (Posada et al. 2002). El nivel de infección de $B$. bassiana tiende a disminuir después de la aplicación, en proporción al tiempo de exposición de las esporas a la radiación solar, más que al efecto de otros factores ambientales o del tipo de formulación (Inglis et al. 1993).

Los resultados de las aplicaciones por aspersión del hongo $B$. bassiana sobre la broca del café para evaluar la patogenicidad en campo son muy variables (Lacayo et al. 1994; Sponagel, 1994; Flórez et al. 1997; Posada et al. 2002) y, posiblemente, obedecen a factores como la tecnología de aplicación, la formulación, las condiciones ambientales de exposición a la radiación solar, la temperatura, la humedad relativa y el momento de aplicación en relación con la emergencia y tránsito de la broca del café.

Los hongos entomopatógenos, además de ser formulados para aplicación por aspersión, se pueden emplear en otras formas, como cebos, pellets y gránulos. Estos tipos de formulación permiten colocar los hongos en trampas que atraen o que brindan refugio a los insectos. Esta respuesta, se ha complementado con el uso de cairomonas o feromonas en las trampas, donde la captura y la muerte del insecto no es más importante que la atracción y paso del insecto por la trampa, que se ha encontrado promisoria para aplicación de organismos entomopatógenos. La utilización de estos métodos persigue aprovechar el comportamiento de los insectos para inocularlos o infectarlos y diseminar los patógenos (Vega et al. 1995; Inglis et al. 1996; Hidalgo et al. 1998; Klein E Lacey, 1999; Smith et al. 1999, Bextine $E$ Thorvilson, 2002; Dow E Vega, 2003; Koller et al. 2005; Hossain et al. 2007; Tinzaara et al. 2007; Arrubla et al. 2008).

Esta forma de aplicación, manipulando a los insectos como vectores, se ha visto como un medio práctico y 
económico para el provecho de los hongos en el control de plagas y enfermedades de plantas. Como insectos vectores, se utilizan tanto plagas como benéficos; dentro de las plagas, insectos de importancia agrícola y de salud pública (Maniania, 2002; Koller et al. 2005, Hossain et al. 2007) y respecto benéficos, como los polinizadores, se utilizan las abejas para transportar el hongo Trichoderma a las flores de plantas, para el control de Botrytis sp., o los abejorros del género Bombus, para dispersar el hongo $B$. bassiana, para el control de moscas blancas en invernaderos (Shafir et al. 2006; Kapongo et al. 2008). Entre las ventajas de este sistema de aplicación de agentes de control biológico: se asegura disminuir la cantidad de inoculo, localizarlo en el sitio apropiado en la cantidad necesaria y, por consiguiente, se contribuye a reducir los costos de aplicación. Adicionalmente, disminuye los riesgos de contaminación y el efecto adverso sobre otros insectos o fauna benéfica

Dentro de la línea de investigación que busca aprovechar las trampas de captura de la broca del café (Cárdenas, 2000; Dufour, 2006) para infectar los adultos y autodiseminar el hongo en el campo, se evaluó y se escogió el sistema de aplicación de las esporas en polvo, porque con este método se obtuvo el mejor resultado de adherencia de las esporas al cuerpo de la broca, en un estudio previo (Arrubla et al. 2008).

Continuando con el avance de esta línea de investigación y por desconocerse el efecto de los factores ambientales sobre la viabilidad y la patogenicidad de las esporas, después de ser aplicadas, en este trabajo se tuvo como objetivo medir la concentración, la pureza, la germinación y la patogenicidad sobre la broca del café de las esporas de $B$. bassiana, aplicadas en polvo en una superficie inerte y expuestas a diferentes condiciones de humedad.

\section{MATERIALES Y MÉTODOS}

El estudio se llevó a cabo en los laboratorios de Entomología del Centro Nacional de Investigaciones del Café (Cenicafé), Chinchiná, Caldas. Se emplearon esporas de Beauveria bassiana (Bals.) Vill. del aislamiento Bb9205, producidas con el método líquidosólido (difásico) de fermentación, en la planta piloto de Cenicafé. Las esporas, se utilizaron en polvo después ser tamizadas $(60$ mallas $(250 \mu \mathrm{m}))$ y secadas, hasta obtener un contenido de humedad $<10 \%$. Previamente, las esporas se mantuvieron almacenadas en disecadores con silica gel, en un cuarto climatizado con $15^{\circ} \mathrm{C}$ y una humedad relativa de $50 \%$ en promedio. Antes de comenzar el experimento, se realizó un control de calidad de las esporas incluyendo evaluaciones de concentración, de pureza, de viabilidad y de patogenicidad hacia $H$. hampei.

Las condiciones ambientales, se establecieron a $24^{\circ} \mathrm{C}$ de temperatura y un ciclo de luz/oscuridad de 12:12 horas. La humedad relativa se obtuvo aplicando soluciones saturadas de $\mathrm{KNO}_{3}, \mathrm{NaCl}+\mathrm{KCl}$, Glucosa y $\mathrm{MgCl}_{6} \mathrm{H}_{2} \mathrm{O}$, que permitieron alcanzar 90, 77, 65 y 39\% de $\mathrm{HR}$, respectivamente (Peterson, 1959; Gilbert et al. 2008). Estas soluciones saturadas, se colocaron en cajas de acrílico de $15 \times 10 \times 5 \mathrm{~cm}$ sin tapa y se cambiaron cada diez días, para conservar constantes los niveles de humedad. Las esporas, se mantuvieron expuestas a los niveles de humedad en "cajas multiusos" de acrilico transparente de $30 \times 20 \times 12 \mathrm{~cm}$. Las cajas, se perforaron lateralmente, para adaptar un termohigrómetro y registrar la humedad.

Las muestras de las esporas, se prepararon para la exposición a las diferentes condiciones de humedad, colocando en cajas de Petri de vidrio sin tapa un gramo de esporas en polvo y cada nivel de humedad relativa tuvo tres cajas o réplicas sin reemplazo. Las cajas de Petri con las muestras de esporas, se colocaron dentro de las "cajas multiusos", correspondientes a cada tratamiento, las cuales, contenían las soluciones saturadas que proporcionaron las humedades antes mencionadas.

Al momento de la evaluación, las esporas removieron y se tomaron tres submuestras de $0,01 \mathrm{~g}$ de las esporas en polvo, expuestas a las diferentes humedades relativas. Cada sub-muestra, se suspendió en $10 \mathrm{~mL}$ de una solución de agua destilada estéril + Tween 80 al $0,1 \%$, obteniendo, de esta forma tres soluciones madres o iniciales, que se emplearon para preparar diluciones consecutivas y se utilizaron para aplicar dos réplicas por submuestra, para determinar las variables: concentración, germinación y pureza. La patogenicidad, se evaluó por inmersión de los adultos de la broca en las suspensiones de esporas, con una concentración de $1 \times 10^{7}$ esporas/mL, obtenida a partir de las soluciones preparadas, previamente. La evaluación de estas variables, se hizo siguiendo la metodología establecida para la evaluación de entomopatógenos por Vélez et al. (1997). 
Para el análisis estadístico de los datos el estudio, se organizó en un diseño completamente al azar. Se evaluaron doce tratamientos, que estuvieron conformados por cuatro niveles de HR $(90,77,65$ y 39\%) y tres de tiempo de exposición: 1, 10, y 20 días y se evaluaron por las variables: concentración, pureza, germinación y patogenicidad; cada tratamiento y variable tuvo seis repeticiones. Los resultados de las variables respuesta concentración y pureza, se analizaron con estadística descriptiva, mientras que las variables germinación y patogenicidad se sometieron al análisis de varianza, con un nivel de significancia del 95\% (SAS Institute, 2003). La patogenicidad, se corrigió con la fórmula de Abbott, porque en el control se presentó mortalidad, ocasionada por otras causas y por hongos, considerados contaminantes (Abbott, 1925).

\section{RESULTADOS Y DISCUSIÓN}

Las pruebas de calidad de las esporas, previo al inicio del experimento, registraron una concentración 9,5 x $10^{10}$ esporas/gramo, pureza $99 \%$, viabilidad $98 \%$ y patogenicidad 93,3\%.

La concentración de las esporas, en los tiempos de evaluación a los 1, 10 y 20, días equivalió a las concentraciones colocadas en las cajas de Petri, al momento de iniciar el experimento. La concentración en las condiciones de la evaluación, se esperaba que no sufriera ninguna alteración, ya que el medio en el que se expusieron las esporas era totalmente inerte; sin embargo, se prevé que cuando se valide la creación de depósitos en las trampas, en condiciones de campo, esta variable puede llegar a sufrir mayores cambios, por el viento y la dinámica de las visitas de las brocas, que van a remover esporas.
La pureza de las esporas ocasionó que en las diferentes evaluaciones la formación de colonias fue superior al $98 \%$ en todos los niveles de humedad. (Tabla 1). En todas las evaluaciones hubo presencia de hongos contaminantes, como: Penicillium, Aspergillus, Fusarium y Levaduras, con porcentajes de unidades formadoras de colonias, menores del 1,0\%. En general, las esporas en polvo evaluadas conservaron una alta pureza, debida, probablemente, a que durante el tiempo de estudio estuvieron confinadas en las cámaras ambientales. La presencia de estos contaminantes en la esporas puedo provenir del proceso de producción y cosecha de las esporas o cuando éstas se depositaron en la cámara de humedad, durante el proceso de manipulación de las muestras.

La germinación fue la variable que mayor variación presentó. Los resultados de germinación disminuyeron en forma gradual y guardaron un rango de variación, entre la mayor y menor germinación de 7,7, 10,4 y 6,0\%, para 1, 10 y 20 días de evaluación, respectivamente. En la primera evaluación, de un día, la germinación logró el mayor valor con $90 \%$ de humedad y disminuyó al decrecer el contenido de esta. En el último lapso conservó valores superiores a 55\%. A los 10 y 20 días de evaluación la tendencia de la germinación sufrió mayor variación. Los valores más altos de germinación, se lograron con los niveles de 39 y $77 \%$ de humedad y las menores con el nivel de $65 \%$.

En el diagnóstico por evaluación (1 día), se presentaron niveles superiores al $80 \%$ de germinación. En el análisis estadístico, se encontró diferencias significativas entre la humedad de $90 \%$ y $39 \%$ ( $\mathrm{GL}=3,39, \mathrm{~F}=4,51 ; \mathrm{P}=$ 0.008). En la segunda evaluación, después de transcurrir diez días, la germinación decreció a niveles entre 79,2

Tabla 1. Porcentaje de pureza de esporas en polvo de B. bassiana expuestas a diferentes niveles de humedad relativa.

\begin{tabular}{|c|c|c|c|}
\hline \multirow{2}{*}{ Humedad \% } & \multicolumn{3}{|c|}{ Evaluación } \\
\cline { 2 - 4 } & 1 día & 10 días & 20 días \\
\cline { 2 - 4 } & Promedio \pm DE & Promedio \pm DE & Promedio \pm DE \\
\hline 90 & $99,9 \pm 0,0$ & $99,9 \pm 0,1$ & $99,8 \pm 0,2$ \\
\hline 77 & $99,9 \pm 0,1$ & $100,0 \pm 0,0$ & $100,0 \pm 0,0$ \\
\hline 65 & $99,9 \pm 0,1$ & $100,0 \pm 0,0$ & $98,8 \pm 0,4$ \\
\hline 39 & $99,0 \pm 0,2$ & $99,4 \pm 0,0$ & $99,5 \pm 0,5$ \\
\hline
\end{tabular}


y $68,2 \%$ y el análisis estadístico reveló diferencias significativas, entre las humedades de 90, 77 y 65\%, y la de 39\%, que alcanzó la mayor germinación (GL = 3, $39, F=10,52 ; P=0.0001)$. En la tercera evaluación, después de 20 días de permanecer, las esporas en los niveles de humedad, la germinación varió entre 62,2 y $56,2 \%$; en los resultados no se encontró diferencias significativas, entre los niveles de humedad $(\mathrm{GL}=3,39$; $\mathrm{F}=2,43 ; \mathrm{P}=0,081)$.

La germinación, se conservó por encima del $50 \%$ al final de los 20 días de evaluación, permitiendo su empleo en trampas con atrayentes, para inocular, infectar y dispersar el hongo, por medio de insectos, como la broca del café. Esto se afirma con base en los resultados previos sobre esta línea de investigación, donde se demostró que la broca puede adquirir, caminando sobre depósitos de esporas en polvo, en menos de un minuto de exposición, hasta 21.556 esporas (Arrubla et al. 2008). Esta cifra garantiza que en la aplicación del hongo en trampas, las brocas que entren en contacto con las esporas y regresen al campo van a ser infectadas, como se demostró en las pruebas de patogenicidad, cuando en todos los tiempos de evaluación la mortalidad estuvo por encima del $86,0 \%$ (Tabla 2). Adicionalmente, hay que tener en cuenta que la broca es un insecto altamente susceptible al hongo $B$. bassiana, ya que cuando entra en contacto, por un minuto con esporas depositadas sobre una superficie tratada con 81 esporas $/ \mathrm{cm}^{2}$, en promedio, es posible tener 82,5 ó 70,0\% de mortalidad, dependiendo de si la formulación aplicada es en aceite o en agua (Posada et al. 2002).

Asegurar $100 \%$ de esporas viables en una aplicación es altamente deseable, con el propósito de asegurar la máxima infección y poder comparar, en igualdad de

Tabla 2. Mortalidad de la broca del café expuesta a esporas en polvo de $B$. bassiana, en diferentes niveles de humedad relativa.

\begin{tabular}{|c|c|c|c|}
\hline \multirow{2}{*}{ Humedad \% } & B. bassiana sin corregir & $\begin{array}{c}\text { B. bassiana + Otras causas } \\
\text { sin corregir }\end{array}$ & B. bassiana corregido Abbott \\
\cline { 2 - 4 } & Promedio $\pm \mathrm{D}: \mathrm{E}$ & Promedio $\pm \mathrm{D}: \mathrm{E}$ & Promedio $\pm \mathrm{D}: \mathrm{E}$ \\
\hline \multicolumn{4}{|c|}{ Evaluación 1 día } \\
\hline 90 & $95,0 \pm 8,4 \mathrm{~A}$ & $96,7 \pm 5,2 \mathrm{~A}$ & $94,8 \pm 8,5 \mathrm{~A}$ \\
\hline 77 & $98,3 \pm 4,2 \mathrm{~A}$ & $100,0 \pm 0,0 \mathrm{~A}$ & $98,3 \pm 4,1 \mathrm{~A}$ \\
\hline 65 & $96,7 \pm 5,2 \mathrm{~A}$ & $100,0 \pm 0,0 \mathrm{~A}$ & $98,3 \pm 4,1 \mathrm{~A}$ \\
\hline 39 & $98,3 \pm 4,1 \mathrm{~A}$ & $100,0 \pm 0,0 \mathrm{~A}$ & $98,3 \pm 4,1 \mathrm{~A}$ \\
\hline Testigo & $0,0 \pm 0,0 \mathrm{~B}$ & $23,3 \pm 15,3 \mathrm{~B}$ & $100,00,0 \pm \mathrm{A}$ \\
\hline \multicolumn{5}{|c|}{ Evaluación 10 días } \\
\hline 90 & $98,3 \pm 4,1 \mathrm{~A}$ & $100,0 \pm 0,0 \mathrm{~A}$ & $100,0 \pm 0,0 \mathrm{~A}$ \\
\hline 77 & $93,3 \pm 5,2 \mathrm{AB}$ & $100,0 \pm 0,0 \mathrm{~A}$ & $94,8 \pm 8,5 \mathrm{~A}$ \\
\hline 65 & $86,7 \pm 10,3 \mathrm{~B}$ & $95,0 \pm 8,4 \mathrm{~A}$ & $100,0 \pm 0,0 \mathrm{~A}$ \\
\hline 39 & $100,0 \pm 0,0 \mathrm{~A}$ & $100,0 \pm 0,0 \mathrm{~A}$ & \\
\hline Testigo & $3,3 \pm 5,7 \mathrm{C}$ & $6,7 \pm 5,8 \mathrm{~B}$ & $96,7 \pm 5,2 \mathrm{~A}$ \\
\hline \multicolumn{5}{|c|}{ Evaluación 20 días } \\
\hline 90 & $96,7 \pm 5,6 \mathrm{~A}$ & $98,3 \pm 4,1 \mathrm{~A}$ & $94,8 \pm 5,7 \mathrm{~A}$ \\
\hline 77 & $90,0 \pm 12,6 \mathrm{~A}$ & $96,7 \pm 5,2 \mathrm{~A}$ & $96,7 \pm 5,2 \mathrm{~A}$ \\
\hline 65 & $95,0 \pm 8,7 \mathrm{~A}$ & $98,3 \pm 4,1 \mathrm{~A}$ & $98,3 \pm 4,1 \mathrm{~A}$ \\
\hline 39 & $98,3 \pm 4,1 \mathrm{~A}$ & $100,0 \pm 0,0 \mathrm{~A}$ & \\
\hline Testigo & $0,0 \pm 0,0 \mathrm{~B}$ & $6,7 \pm 5,8 \mathrm{~B}$ & \\
\hline
\end{tabular}

* Promedios seguidos por la misma letra, en las columnas, no presentan diferencia significativa (Prueba de Duncan P $\leq 0,05)$. 
condiciones, respuesta a concentraciones (Ugine et al. 2005); sin embargo, en el caso de la aplicación de esporas en depósitos, sujetos al efecto inmediato de los factores ambientales, no es tan exigente contar con esporas con $100 \%$ de viabilidad; incluso en el proceso de producción es difícil obtener esporas $100 \%$ viables. Para cumplir con este requisito, se necesitaría adicionar mayor cantidad de esporas, lo que resultaría en un costo extra.

Los resultados de la germinación indican que no es preciso hacer correcciones para tener esporas $100 \%$ viables, puesto que por los estimativos presentados previamente, se puede recomendar el uso de las esporas con niveles de germinación superiores a $50 \%$, depositadas en una superficie inerte, por un tiempo hasta de 20 días. Esta recomendación de aplicación obedece al conocimiento que se tiene del comportamiento de la broca de entrar en contacto con el hongo, de adquirir esporas y de conocer la cantidad de esporas necesarias que se requieren para infectar la broca y causar su muerte (Cl50) (Posada et al. 2002; Arrubla et al. 2008).

La patogenicidad para todas las evaluaciones causó una mortalidad de la broca del café superior al $85 \%$. En la primera evaluación, la mortalidad superó el 95\%, mientras que en la segunda, el valor más bajo, $86,7 \%$, para todo el experimento, se presentó en el tratamiento de $65 \%$ de humedad. En la tercera evaluación, la mortalidad para todos los tratamientos fue superior al 90\% (Tabla 2).

En las valoraciones, se encontró mortalidad de la broca del café sin signos del hongo $B$. bassiana y brocas con crecimiento de hongos considerados contaminantes. A este conjunto de mortalidad, se le denominó muerte por otras causas. La mayor mortalidad por otras causas, se presentó en el testigo de la primera evaluación (23,3\%), donde la presencia de brocas que mostraron crecimiento de hongos contaminantes, alcanzó 11,7\%; los hongos contaminantes, se identificaron como Penicillium, Aspergillus, Fusarium. La presencia de ellos, tanto en las esporas (Tabla 1) como en las evaluaciones de patogenicidad (Tabla 2), alcanzaron niveles de $10 \%$, que es considerado como el máximo tolerable de mortalidad, por otras causas en bioensayos.

Respecto a la presencia de contaminantes sobre la broca, se conoce que existe una alta cantidad y diversidad de microorganismos, que aparecen como contaminantes en los bioensayos de pruebas de patogenicidad (Posada et al. 1998; Posada et al. 2002). La presencia de hongos contaminantes sobre la broca, probablemente, se debe a que éstos están presentes en el interior (intestino) o adheridos al integumento del insecto (Pérez et al. 2003). La broca obtiene estos microorganismos contaminantes durante el proceso de cría en las cerezas de café, a pesar de los procesos de desinfectación del grano y de la broca.

En las pruebas de patogenicidad cuando la broca muere por causas naturales o por acción de los entomopatógenos, los contaminantes crecen como saprofitos (Posada et al. 1998; Posada et al. 2002). Se sospecha que su presencia y su crecimiento, se debe a las condiciones favorables del estudio y, que crecen simultáneamente con los hongos entomopatógenos y, en algunos casos, son completamente dominantes y no se puede encontrar el hongo entomopatógeno, que se está evaluando.

Lo anterior puede conducir a descalificar a los hongos entomopatógenos y la propuesta de utilizar las trampas para la auto-diseminación de las esporas del hongo $B$. bassiana. Para evitar que esto ocurra, se debe conocer, apropiadamente, estos contaminantes y tomar las medidas de asepsia en la producción de las esporas, en la ejecución de los bioensayos y en la selección del material biológico (esporas y la broca dependiendo del método de cría), para prevenir que tomen ventaja y parezcan como dominantes. Todavía, se desconoce el nivel de presencia de los contaminantes que puede afectar la eficiencia de infección de la broca por competencia con el hongo $B$. bassiana o que puede llegar a aparecer sobre los cadáveres de la broca de realizarse pruebas de patogenicidad con brocas capturadas en trampas con atrayentes y que, a su vez, se vayan a utilizar para dispersar el hongo en los cafetales.

Los resultados indican que las esporas de $B$. bassiana en polvo, expuestas a los niveles de humedad relativa por un tiempo de 20 días conservaron la patogenicidad con niveles altos, superior al $86 \%$, en condiciones de laboratorio $\left(24^{\circ} \mathrm{C}\right.$ en promedio) (Tabla 2). Estos resultados señalan que las esporas de $B$. bassiana se pueden utilizar con seguridad, ya que la humedad relativa no afectó la patogenicidad de las esporas por el tiempo de evaluación y dan la oportunidad de poder ser 
empleadas en ambientes, como el de la zona cafetera, donde predominan las condiciones evaluadas de temperatura y de humedad relativa (Guzmán \& Baldión, 2003).

La tendencia de conservación de la germinación y de la patogenicidad de las esporas con valores superiores al 55 y $86 \%$, respectivamente, por 20 días y los criterios analizados de concentración y pureza (Figura 1, Tabla 2), estimula considerar el uso de trampas con atrayentes de la broca, y que contengan depósitos de esporas, que, simultáneamente, permitan la infestación de la broca. Esta metodología validada en campo y de resultar exitosa puede llegar a ampliar el uso de métodos de aplicación de los hongos entomopatógenos. Adicionalmente, permite hacer un empleo racional y ahorro de recursos, como son las esporas y el tiempo de labores para mantener las trampas, ya que regularmente hay que cambiar y colocar nuevos depósitos de esporas infectivas contra la broca del café.

Se prevé que este sistema de aplicación del hongo en trampas y el uso de la broca para diseminar el hongo es altamente promisorio, puesto que garantiza el mantenimiento de una población alta de inoculo (esporas), no solo en las trampas sino también reciclando en el campo sobre los cadáveres de la broca, los cuales, se ha estimado que pueden producir hasta $1 \times 10^{6}$ esporas por broca (Narváez et al. 1997; Posada E Vega, 2005). Este modelo de manipulación del hongo puede ahorrar costos de aplicación, con equipos convencionales, donde las esporas quedan expuestas a los factores ambientales que deterioran, rápidamente, su calidad (Inglis et al. 1993, 1995), permite el uso de menos cantidades de hongo y se constituye en un método agro-ecológico de gran impacto socio-económico para los productores del grano, que poseen limitaciones de mano de obra y recursos financieros.

Adicional a esto, es importante determinar que el tiempo de aplicación corresponda con el tiempo de máxima actividad (tránsito) de la broca del café. En la aspersión en campo, se busca que la aplicación asegure el óptimo cubrimiento y la infección del insecto; sin embargo, esto no se logra, generalmente, por las condiciones del terreno que no permiten el tratamiento en una forma rápida, ya que los equipos empleados en el cultivo del café y la topografía del terreno (Waller et al. 2007) no

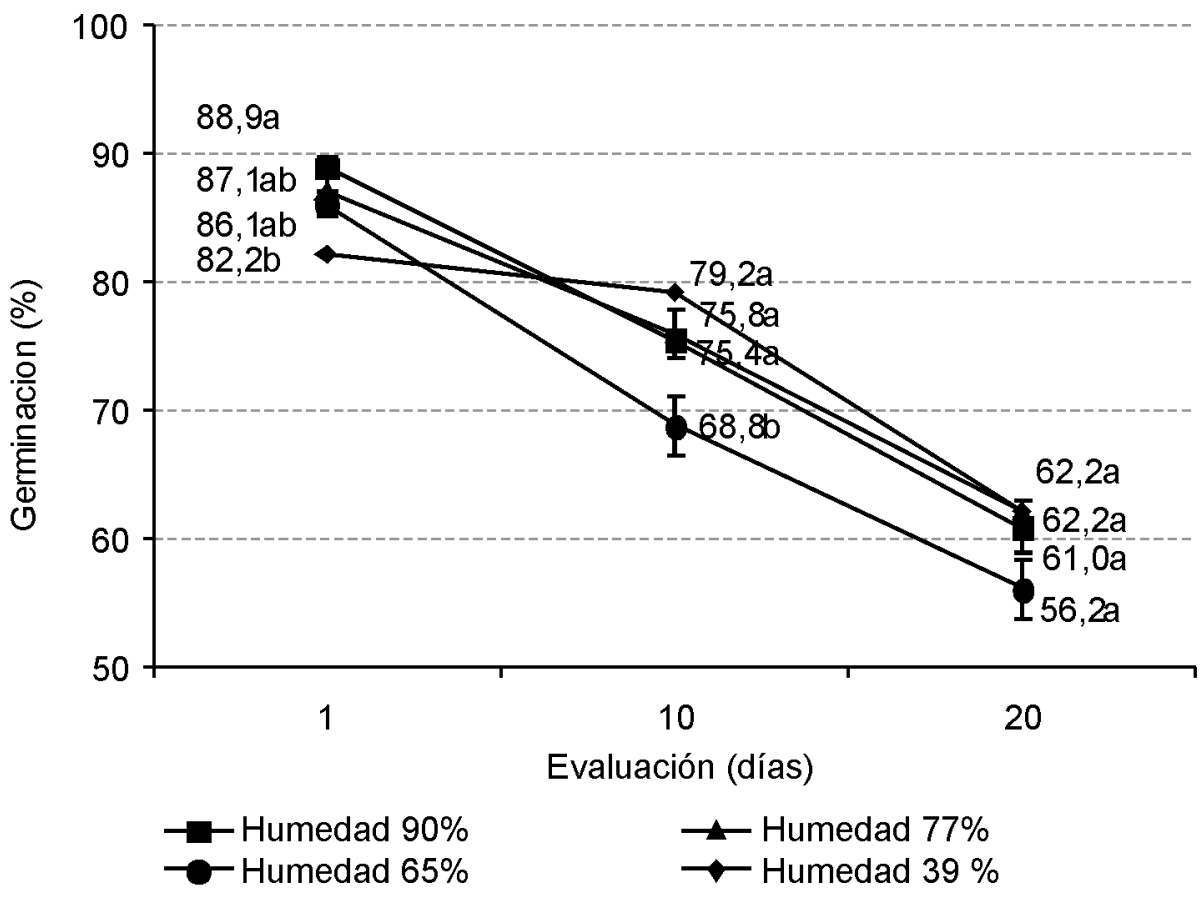

Figura 1. Germinación de las esporas de B. bassiana, a través del tiempo, bajo diferentes niveles de humedad relativa. 
permiten obtener un buen cubrimiento o depósito de gotas con esporas. Es por esto que la propuesta de utilizar diseños de trampas con atrayentes y que protejan los depósitos de esporas de los factores ambientales, se considera que puede llegar a contribuir a hacer el mejor uso de las esporas, como insumo biológico, al garantizar su viabilidad e infectividad, por un largo tiempo (Figura 1, Tabla 2). El próximo paso en esta línea de investigación es validar los resultados en campo y medir el efecto de los factores ambientales, incluido la temperatura, la humedad, la radiación y el viento.

AGRADECIMIENTOS: Los autores expresan sus agradecimientos a Eduardo Osorio, por su colaboración en la ejecución de los experimentos. A Cenicafé y Conciencias, por la financiación. A Mónica Pava-Ripoll, por revisiones a este manuscrito. Conflictos de intereses: Esta investigación y el manuscrito fueron realizados por todos los autores, por tanto, declaramos que no existe ningún conflicto de intereses que ponga en riesgo la validez de la presente publicación.

\section{BIBLIOGRAFÍA}

1. ABBOTT, W.S. 1925. A method of computing the effectiveness of an insecticide. J. Econ. Entomol. 18:265-267.

2. ARRUBLA M., P.F.; CÁRDENAS R., M.; POSADA F., F.J. 2008. Adherencia de las esporas de Beauveria bassiana formuladas en polvo y líquido sobre la broca del café. Rev. U.D.C.A Act. E Div. Cient. (Colombia). 11(1):123-131.

3. BATEMAN, R.P.; CAREY, M.; MOORE, D.; PRIOR, C. 1993. The enhanced infectivity of Metarhizium flavoviride in oil formulations to desert locusts at low humidities. Ann. Appl. Biol. 122:145-152.

4. BEXTINE, B.; THORVILSON, H.G. 2002. Field Applications of bait-formulated Beauveria bassiana alginate pellets for biological control of the red imported fire ant (Hymenoptera: Formicidae). Environ. Entomol. 31(4):746-752.

5. CÁRDENAS M., R. 2000. Trampas y atrayentes para monitoreo de poblaciones de broca del café Hypothenemus hampei (Ferrari) (Col., Scolytidae). In: SIMPOSIO Latinoamericano de Caficultura, 19.
San José (Costa Rica), Octubre 2-6, 2000. Memorias. San José (Costa Rica), ICAFE-PROMECAFE. p.369-379.

6. DOWD, P.F.; VEGA, F.E. 2003 Autodissemination of Beauveria bassiana by sap beetles (Coleoptera: Nitidulidae) to overwintering sites. Biocontrol Sci. Techn. 13(1):65-75.

7. DUFOUR, B.P. 2006. Elaboración de un método estándar para la evaluación del trampeo de la broca del café Hypothenemus hampei Ferr. Bol. Promecafé (Costa Rica). 109:5-9.

8. FERRON, P. 1978. Biological control of insect pests by entomogenous fungi. Ann. Rev. Entomol. 23:409-442.

9. FLÓREZ M., E.; BUSTILLO P., A.E.; MONTOYA R., E.C. 1997. Evaluación de equipos de aspersión para el control de Hypothenemus hampei con el hongo Beauveria bassiana. Cenicafé (Colombia). 48(2):92-98.

10. GILBERT, J.; WOODS, S.M.; KROMER, U. 2008. Germination of ascospores of Gibberella zeae after exposure to various levels of relative humidity and temperature. Phytopathology. 98:504-508.

11. GUZMÁN M., O.; BALDIÓN R., J.V. 2003. El clima en la sede principal del Centro Nacional de Investigaciones de Café, Chinchiná, Caldas. Cenicafé (Colombia) 54(2):110-133.

12. HIDALGO, E.; MOORE, D.; LE PATOUREL, G. 1998. The effect of different formulation of Beauveria bassiana on Sitophilus zeamais in stored maize. J. Stored Prod. Res. 34(2-3):171-179.

13. HOSSAIN, M.S.; WILLIAMS, D.G.; HOSSAIN, M.A.B.M.; NORNG, S. 2007. Comparison of trap designs for use with aggregation pheromone and synthetic co-attractant in a user-friendly attract and kill system to control Carpophilus spp. (Coleoptera: Nitidulidae). Austral. J. Entomol. 46: 244-250.

14. INGLIS, G.D.; GOETTEL, M.S.; JOHNSON, D.L. 1993. Persistence of the entomopathogenic 
fungus, Beauveria bassiana, on phylloplanes of crested wheatgrass and alfalfa. Biol. Control. 3:258-270.

15. INGLIS, G.D.; JOHNSON, D.L.; GOETTEL, M.S. 1995. Influence of ultraviolet light protectans on persistence of the entomopathogenic fungus, Beauveria bassiana. Biol. Control. 5:581-590.

16. INGLIS, G.D.; JOHNSON, D.L.; GOETTEL, M.S. 1996. Effect of bait substrate and formulation on infection of grasshopper nymphs by Beauveria bassiana. Biocontrol Sci.Techn. 6:35-50.

17. KAPONGO, J.P.; SHIPP, L.; KEVAN, P.; BROADBENT, B. 2008. Optimal concentration of Beauveria bassiana vectored by bumble bees in relation to pest and bee mortality in greenhouse tomato and sweet pepper. Biol. Control. 53(5):797-812.

18. KLEIN, M.G.; LACEY, L.A. 1999. An attractant trap for autodissemination of entomopathogenic fungi into population of the Japanese beetles Popillia japonica (Coleoptera: Scarabaeidae). Biocontrol Sci. Techn. 9:151-158.

19. KOLLER, R.; JUNG, K.; SCHEU, S.; ZIMMERMANN, G.; RUTHER, J. 2005. Biocontrol of the forest cockchafer (Melolontha chafer hippocastani): Experiments on the applicability of the "Catch and Infect"- Technique using a combination of attractant traps with the entomopathogenic fungus Beauveria brongniartii. IOBC/wprs Bull. 28(2):37-44.

20. LACAYO, L.; BARRIOS, M.; JIMÉNEZ, C.; SANDINO, V. 1994. Uso de hongos entomopatógenos para el manejo de la broca del café (Hypothenemus hampei) en Nicaragua. Ministerio de Agricultura y Ganadería, Nicaragua, MAG Proyecto CATIE INTA / MIP. 5p.

21. LISANSKY, S.G.; COOMBS, J. 1994. Developments in the market for biopesticides. Brighton Crop Protection Conference - Pests and Diseases. 1:1049-1054.

22. MANIANIA, N.K. 2002. A low-cost contamination device for infecting adult Tsetse Flies, Glossina spp., with the entomopathogenic fungus Metarhizium anisopliae in the Field. Biocontrol Sci. Techn. 12:59- 66.

23. MARQUEZ, E.J.; ALVES, S.B. 1996. Optimization of formulations on the preservation of conidia of Beauveria bassiana (Bals.) Vuill. and Metharhizium anisoplae (Metschn.) Sorok. under different conditions of storage. Arquivos de Biologia e Tecnología. 39:861-877.

24. McCOY, C.W.; SAMSON, R.A.; BOUCIAS, D.G. 1988. Entomogenous fungi. p. 151-236. In: IGNOFFO, C.M. Ed. Handbook of Natural Pesticides, Vol. 5, Microbial Insecticides, Part A, Entomopathogenous Protozoa and Fungi. CRC Press, Boca Raton, Florida. 243p.

25. MOORE, D.; BRIDGE, P.D.; HIGGINS, P.M.; BATEMAN, R.P.; PRIOR, C. 1993. Ultra-violet radiation damage to Metharizium flavoviride conidia and the protection given by vegetable and mineral oils and chemical sunscreens. Ann. Appl. Biol. 122:605-616.

26. MOORE, D.; BATEMAN, R.P., CAREY, M.; PRIOR, C. 1995. Long-term storage of Metarhizium flavoviride conidia in oil formulations for the control of locusts and grasshoppers. Biocontrol Sci. Techn. 5:193-199.

27. NARVÁEZ G., M. del P.; GONZÁLEZ G., M.T.; BUSTILLO P., A.E.; CHAVES C., B.; MONTOYA R., E.C. 1997. Producción de esporas de los hongos Beauveria bassiana y Metarhizium anisopliae cultivados en arroz y sobre la broca del café. Rev. Col. Entomol. 23(2-3):125-131.

28. PÉREZ, J.; INFANTE, F.; VEGA, F.E.; HOLGUÍN, F.; MACÍAS, J.; VALLE, J.; NIETO, G.; PETERSON, S.W.; KURTZMAN, C.P.; O'DONNELL, K. 2003. Mycobiota associated with the coffee berry borer (Hypothenemus hampei) in Mexico. Mycol. Res. 107:879-887.

29. PETERSON, A. 1959. Entomological techniques. How to work with insects. Ed. Edwards Brotheres. Michigan.435p. 
30. POSADA F., F.J.; MARÍN M., P.; PÉREZ S., M. 1998. Paecilomyces lilacinus, enemigo natural de adultos de Hypothenemus hampei. Cenicafé (Colombia). 49(1):72-77.

31. POSADA F., F.J.; OSORIO, V.E.; VELÁZQUEZ S., E.T. 2002. Evaluación de la patogenicidad de Beauveria bassiana sobre la broca del café empleando el método de aspersión foliar. Rev. Col. Entomol. 28(2):139-144.

32. POSADA, F.; VEGA, F. 2005. A new method to evaluate the biocontrol potential of single spore isolates of fungal entomopathogens. 10p. J. Insect Science. 5:37. Disponible desde Internet en: insectscience.org/5.37 (con acceso el 09/10/09).

33. SAS, INSTITUTE. 2003. SAS/Stat. User's guide. SAS Institute Inc., Cary, NC. 1028p.

34. SHAFIR, S.; DAG, A.; BILU, A.; ABU-TOAMY, M.; ELAD, Y. 2006. Honey bee dispersal of the biocontrol agent Trichoderma harzianum T39: effectiveness in suppressing Botrytis cinerea on strawberry under field conditions. Europ. J. Plant Pathology. 116(2):119-128.

35. SMITH, S.M.; MOORE, D.; KARANJA, L.W.; CHANDI, E.A. 1999. Formulation of vegetable fat pellets with pheromone and Beauveria bassiana to control the larger grain borer, Prostephanus truncatus (Horn.). Pest. Sci. 55:711-718.

36. SPONAGEL, K.W. 1994. La broca del café Hypothenemus hampei en plantaciones de café robusta en la Amazonía Ecuatoriana. Wissenschaftlicher Fachverlag. Giessen. Alemania. 185p.

37. TINZAARA, W.; GOLD, C.S.; DICKE, M.; VAN HUIS, A.; NANKINGA, C.M.; KAGEZI, G.H.; RAGAMA,
P. 2007. The use of aggregation pheromone to enhance dissemination of Beauveria bassiana for the control of the banana weevil in Uganda. Biocontrol Sci. Techn. 17(2):111-124.

38. UGINE, T.A.; WRAIGHT, S.P.; BROWNBRIDGE, M.; SANDERSON, J.P. 2005. Development of a novel bioassay for estimation of median lethal concentrations (LC50) and doses (LD50) of the entomopathogenic fungus Beauveria bassiana, against western flower thrips, Frankliniella occidentalis. J. Invertebr. Path. 89:210-218.

39. VEGA, F.E.; DOWD, P.F.; BARTELT, R.J. 1995. Dissemination of microbial agents using an autoinoculating device and several insect species as vectors. Biol. Contr. 5:545-552.

40. VÉLEZ A., P.E. 1997. Evaluación de formulaciones en aceite y agua del hongo Beauveria bassiana (Balsamo) Vuillemin en campo. Rev. Col. Entomol. 23(1-2):137-142.

41. VÉLEZ A., P.E.; MONTOYA R., E.C. 1993. Supervivencia del hongo Beauveria bassiana bajo radiación solar en condiciones de laboratorio y campo. Cenicafé (Colombia). 44(3):111-122.

42. VÉLEZ A., P.E.; POSADA F., F.J.; MARÍN, P.; BUSTILLO P., A.E.; GONZALES G., M.T.; OSORIO, E. 1997. Técnicas para el control de calidad de formulaciones de hongos entomopatógenos. Boletín Técnico, No 17. Cenicafé (Colombia). 37p.

43. WALLER, J.M.; BIGGER, M.; HILLOCKS, R.J. 2007. Coffee pests, diseases and their management. Wallingford (Inglaterra), CAB. 434p.

Recibido: Agosto 27 de 2009

Aceptado: Febrero 19 de 2010 